\title{
Design of an experimental apparatus for measurement of the surface tension of metastable fluids
}

\author{
V. Vinš ${ }^{1, a}$, J. Hrubý ${ }^{1}$, J. Hyk1 $1^{1}$, J. Blaha ${ }^{1}$ and B. Šmíd ${ }^{1}$ \\ ${ }^{1}$ Institute of Thermomechanics AS CR, v.v.i., Dolejškova 1402, 18200 Prague 8, Czech Republic ( ${ }^{a}$ vins@it.cas.cz)
}

\begin{abstract}
A unique experimental apparatus for measurement of the surface tension of aqueous mixtures has been designed, manufactured, and tested in our laboratory. The novelty of the setup is that it allows measurement of surface tension by two different methods: a modified capillary elevation method in a long vertical capillary tube and a method inspired by the approach of Hacker (National Advisory Committee for Aeronautics, Technical Note 2510, 1-20, 1951), i.e. in a short horizontal capillary tube. Functionality of all main components of the apparatus, e.g., glass chamber with the capillary tube, temperature control unit consisting of two thermostatic baths with special valves for rapid temperature jumps, helium distribution setup allowing pressure variation above the liquid meniscus inside the capillary tube, has been successfully tested. Preliminary results for the surface tension of the stable and metastable supercooled water measured by the capillary elevation method at atmospheric pressure are provided. The surface tension of water measured at temperatures between $+26^{\circ} \mathrm{C}$ and $-11^{\circ} \mathrm{C}$ is in good agreement with the extrapolated IAPWS correlation (IAPWS Release on Surface Tension of Ordinary Water Substance, September 1994); however it disagrees with data by Hacker.
\end{abstract}

\section{Introduction}

Surface tension is an important thermophysical property playing role in many natural, environmental, and technical processes. Even though, this property has been relatively well described for most of the commonly used industrial fluids, e.g. for water, hydrocarbons, and various mixtures, the available experimental data are still rather scarce in some temperature regions. Moreover, most of the data are limited to the thermodynamically stable conditions. In this study, we focus on the surface tension in the metastable state, i.e. on the cases when the supercooled liquid is cooled down below the freezing temperature. The surface tension of supercooled liquids plays an important role in the nucleation of water droplets and ice in the upper atmosphere, aircraft icing, and condensation shocks in supersonic flow [1].

A unique experimental apparatus for measurement of the surface tension of water and binary aqueous mixtures is described in this study. The basic measuring method is inspired by the approach by Floriano and Angell [2]. The surface tension of the metastable supercooled water can be measured using a modified capillary elevation method when the upper meniscus inside a vertical capillary tube is cooled down, while a liquid column below the meniscus together with a water container can be left at an ambient temperature. Height of the liquid column inside the capillary tube is driven by the surface tension which varies with temperature of the upper meniscus.

Design of the experimental apparatus allows its modification also for measurement of surface tension for supercooled water on the basis of a method developed by Hacker [1]. In this case, the liquid thread is located inside a short horizontal capillary tube whose one end is connected to the setup allowing pressure variation. The second end of the tube is opened to the ambient. The surface tension of investigated liquid can be evaluated from the pressure required for flattening the liquid meniscus at the opened end of the capillary tube.

Results for the surface tension of pure water presented in this study have been measured with the elevation method inside a vertical capillary tube.

Pure water has been prepared from two serially connected cleaning devices. Reverse osmosis unit Rowapur $^{\mathrm{A}}$ can be connected to the common water distribution system and removes up to $98 \%$ of dissolved impurities. The output water from Rowapur is subsequently cleaned inside Neptune Analytical ${ }^{\mathrm{B}}$ device which provides ultrapure water with guaranteed resistivity $18.2 \mathrm{M} \Omega \cdot \mathrm{cm}$ and total organic carbon (TOC) less than $1 \mathrm{ppb}$ (parts per billion).

\footnotetext{
${ }^{\text {A DeltaChrom }}{ }^{\mathrm{TM}}$, Watrex Praha, s.r.o.; http://www.watrex.cz/

${ }^{B}$ Purite Limited; http://www.purite.com/
} 


\section{Design of an experimental apparatus}

The surface tension measured by the capillary elevation method can be determined from the hydrostatic pressure of elevated liquid column $\Delta p$ and inner diameter of the capillary tube $d$. The hydrostatic pressure can be determined from the measured height of the liquid column $h$ and density of the liquid $\rho$ at a given temperature ( $\Delta p=h \rho g$ ).

$$
\sigma=\frac{\Delta p d}{4}=\frac{h \rho g d}{4}
$$

Height of the liquid column, i.e. distance between the upper meniscus inside the capillary tube and the level in the liquid container, was measured manually with the cathetometer. The uncertainty of the measured height was $\pm 0.04 \mathrm{~mm}$. Besides the manual measurement, the meniscus inside the capillary tube can also be observed with a high resolution camera Basler ${ }^{\mathrm{C}}$ pilot piA 2400 connected to a PC. With an approximate inner diameter of the capillary tube $0.3 \mathrm{~mm}$, the water column elevates to $9 \mathrm{~cm}$ to $10 \mathrm{~cm}$ under standard conditions.

The capillary tube was cleaned with chromosulfuric acid and pure water before the measurement.

\subsection{Glass chamber with the capillary tube}

A special in house-made glass chamber with the glass capillary tube represent main parts of the experimental apparatus. The cylindrical glass chamber is connected with a set of optical glasses on its bases to two additional chambers. These two smaller chambers are evacuated or filled with a dry gas before the measurement to avoid condensation of air humidity on the optical glasses at temperatures below the dew point of ambient air.

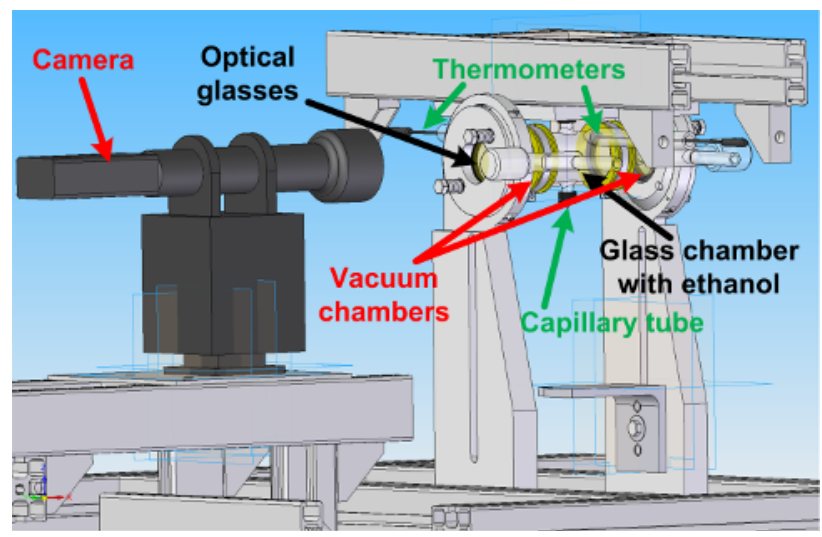

Fig. 1. Scheme of the glass chamber with the capillary tube

Figure 1 shows a 3D design of the glass chamber with the capillary tube, vacuum chambers, and a camera observing the liquid meniscus. All three chambers together with connecting tubing are insulated with thermal foam Armaflex ${ }^{\mathrm{D}}$ insulation.

\footnotetext{
${ }^{\mathrm{C}}$ Basler AG; http://www.baslerweb.com/

${ }^{D}$ Armacell LLC; http://www.armacell.com/
}

\subsection{Temperature control unit}

Unlike in case of Floriano and Angell [2] who used nitrogen flow for varying the temperature of the meniscus inside the capillary tube, the glass chamber with the capillary tube has been connected to two thermostatic baths with liquid ethanol in this study. Liquid ethanol flowing through the chamber assures significantly higher heat transfer and consequently better temperature regulation than gaseous nitrogen. Two thermostatic baths; namely Haake ${ }^{\mathrm{E}}$ C50 and Lauda ${ }^{\mathrm{F}} R P$ 855, can operate at temperatures down to $-45^{\circ} \mathrm{C}$. The first thermostatic bath operates at an ambient temperature and the second one is set to the desired measuring temperature. Figure 2 shows simplified scheme of the ethanol distribution unit equipped with two special switch-valves of own design that allow quick connection of the glass chamber to the relevant thermostatic bath. The entire ethanol distribution unit can also be used on simultaneously designed test stand for measurement of the density of supercooled water [3].

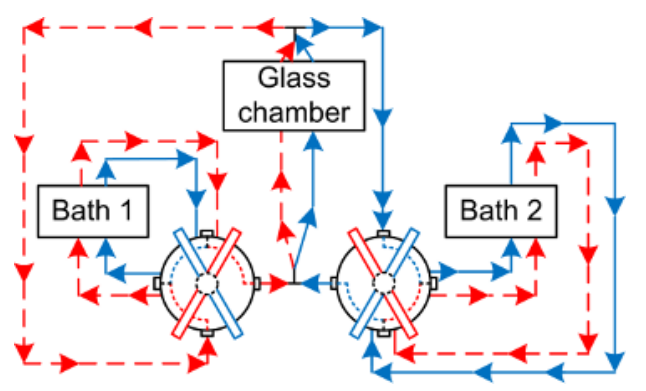

Fig. 2. Scheme of the thermostatic baths connected to the glass chamber

Temperature of the ethanol flowing inside the glass chamber was measured by two precise resistive thermometers Pt100 from Omega ${ }^{\mathrm{G}}$ manufacturer placed in the position close to the capillary tube. The thermometers connected to the digital thermometer bridge $A S L^{\mathrm{H}} F 500$ were calibrated in the temperature range from $-30{ }^{\circ} \mathrm{C}$ to $+30{ }^{\circ} \mathrm{C}$. Standard uncertainty of the measured temperature was below $\pm 0.01 \mathrm{~K}$.

\subsection{Helium distribution setup}

The upper end of the capillary tube has been connected to the helium distribution setup. Water inside the capillary tube is not in contact with ambient air containing potential pollutants but with clean helium in this case. The water meniscus shall therefore not be affected on one hand by aerosol impurities and on the other hand by dissolved gases, since helium has much lower solubility in water than air. The adsorption of helium on the liquid surface is also negligible. The pressure above the water meniscus inside the capillary tube can be changed by varying a flow rate of helium through the distribution setup shown in figure 3. The helium setup allows fine

\footnotetext{
E Thermo Haake GmbH ; http://www.thermohaake.de/

${ }^{\mathrm{F}}$ Lauda Dr. R. Wobser GmbH \& Co.KG; http://www.lauda.de/

${ }^{\mathrm{G}}$ Omega Engineering, INC.; http://www.omega.com/

${ }^{\mathrm{H}}$ Automatic Systems Laboratories; http://www.aslltd.co.uk/
} 
setting of the pressure above the liquid meniscus together with flushing the capillary tube with helium before the measurement.

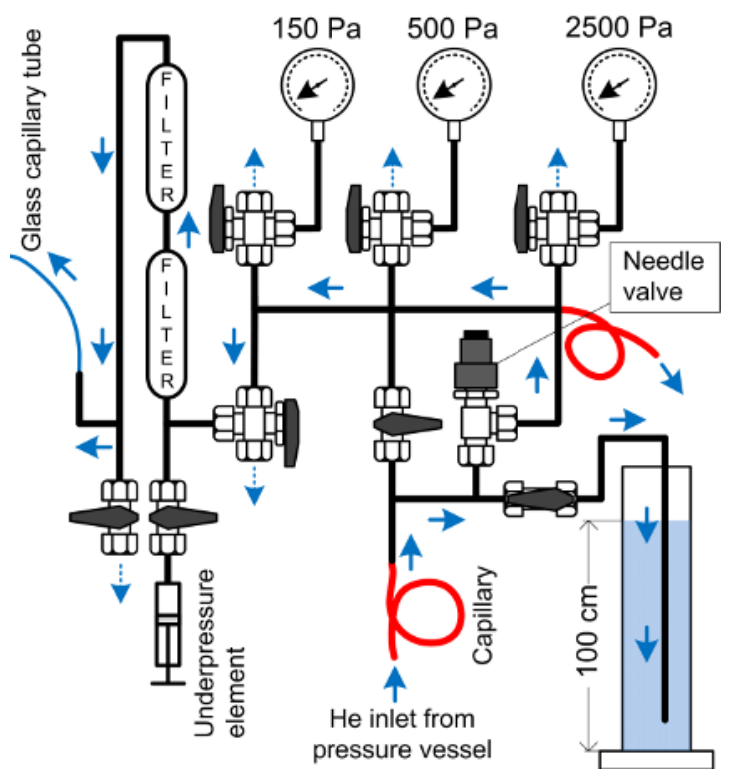

Fig. 3. Scheme of the helium distribution setup

Helium is supplied from a gas cylinder through a stainless steel capillary tube with an inner diameter of $0.25 \mathrm{~mm}$ and length of $350 \mathrm{~mm}$. The stainless steel capillary tube represents a hydrodynamic resistance and consequently the flow rate of helium through the entire system is reduced to $200 \mathrm{sccm}$ (standard cubic centimeters per minute) at a fixed pressure 5 bar at the capillary tube inlet. The throttled gas coming from the capillary tube is distributed between a high precision needle valve and a horizontal cylinder with a water column of 1 meter height. The pressure at the entrance to the metering valve has a constant value given as a sum of the atmospheric pressure and the hydrostatic pressure of the water column in the horizontal cylinder, i.e. $\mathrm{p} \approx 111.1 \mathrm{kPa}$. The metering needle valve Parker $^{\mathrm{I}}$ with low flow coefficient $\mathrm{Cv}=0.0004$ serves for tuning the flow rate of helium in the section connected to the glass capillary tube. The pressure inside the glass capillary tube is given by the pressure drop at a short stainless steel capillary tube with an exhaust to the atmosphere. The capillary tube has an inner diameter of $0.25 \mathrm{~mm}$ and length of $20 \mathrm{~mm}$. The estimated flow rate through this capillary tube shall not exceed $150 \mathrm{sccm}$. An underpressure element represented by a $2 \mathrm{ml}$ syringe is connected to the system to allow an additional elevation of the water column inside the glass capillary tube.

Pressure of helium inside the section connected to the glass capillary tube can be measured by three differential pressure sensors Furness Controls ${ }^{\mathrm{J}}$ FCO332 with pressure ranges of $150 \mathrm{~Pa}, 500 \mathrm{~Pa}$, and $2500 \mathrm{~Pa}$ and accuracies of $\pm 0.05 \mathrm{~Pa}, \pm 0.30 \mathrm{~Pa}$, and $\pm 1.80 \mathrm{~Pa}$, respectively. Organic impurities and aerosol particles can strongly affect behavior of the phase interface inside the

\footnotetext{
I Parker Hannifin Corp.; http://www.parker.com/

${ }^{\mathrm{J}}$ Furness Controls Ltd.; http://www.furness-controls.com/
}

capillary tube and consequently the value of measured surface tension. Therefore, the system is equipped with two filters placed before the glass capillary tube. These filters shall avoid helium contamination by organic (filter Restek $^{\mathrm{K}} \quad$ capillary-grade hydrocarbon trap) and mechanical (Swagelok ${ }^{\mathrm{L}} F W$ series filter) impurities.

The control of the pressure inside the upper part of the glass capillary tube allows keeping the liquid meniscus at desired position. This fact represents an important advantage compared to the original method by Floriano and Angell [2] because an error due to possible variation of the capillary diameter is avoided. However, the data presented in this study were measured by a standard elevation method without pressure compensation. The helium distribution setup is operational, however there are still few details which require further attention, e.g., tuning of the camera observing the meniscus inside the capillary tube, proper settings of the inlet pressure from the pressure vessel, i.e. flow rate though the entire system, and height of the water column inside the graduated cylinder, i.e. pressure before the metering needle valve.

\section{Preliminary experimental results}

Successful preliminary tests of the entire apparatus have been performed during year 2012 in our laboratory. Figure 4 shows an example of the measured results. The water meniscus inside the glass capillary tube was observed with the digital camera at two different temperatures of $+18^{\circ} \mathrm{C}$ and $-9^{\circ} \mathrm{C}$. Temperature drop of $27^{\circ} \mathrm{C}$ corresponds to the height increase of the water column of about $0.5 \mathrm{~cm}$.

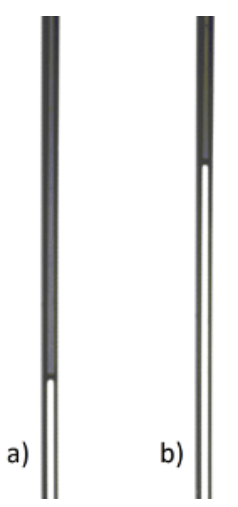

Fig. 4. Water meniscus inside a glass capillary tube observed by a digital camera a) at $+18{ }^{\circ} \mathrm{C}$, b) at $-9^{\circ} \mathrm{C}$

\subsection{Analysis of measured data}

The surface tension of stable and supercooled water was determined from the measured height of the water column inside the glass capillary tube $h$, temperature inside the glass chamber $T$, and the ambient temperature $T_{\mathrm{AMB}}$. The temperature inside the glass chamber stabilized during five to ten minutes after the switchvalves on the temperature control unit were switched

\footnotetext{
${ }^{\mathrm{K}}$ Restek; http://www.restek.com/

${ }^{\mathrm{L}}$ Swagelok; http://www.swagelok.com/
} 
over. Similarly as in the study of Floriano and Angell [2], the measurement was performed with a descending meniscus. After equilibration of temperature inside the glass chamber, the water column in the capillary tube was pulled up by reducing the helium pressure above the meniscus with the underpressure element; see figure 3. The helium distribution setup was then opened to atmosphere and the meniscus descended to the equilibrium position.

The inner diameter of the capillary tube $d$ was evaluated from the measurements performed at temperatures above $0.01{ }^{\circ} \mathrm{C}$.

$$
d=\frac{4 \sigma_{\text {corr }}}{\Delta p}=\frac{4 \sigma_{\text {corr }}}{g\left(\rho_{\mathrm{IN}} h_{\mathrm{IN}}+\rho_{\mathrm{AMB}} h_{\mathrm{AMB}}\right)}
$$

In equation (2), $h_{\mathrm{IN}}$ denotes height of the water column having temperature $T$ inside the glass chamber, $h_{\mathrm{AMB}}$ is the height of the water column outside the glass chamber at the ambient temperature $T_{\mathrm{AMB}}, g$ stands for local gravity $\left(9.80993 \mathrm{~m} \cdot \mathrm{s}^{-2}\right), \rho$ is the density of water at a given temperature, and $\sigma_{\text {corr }}$ is the correlated surface tension of water at temperature $T$. The correlated surface tension was given by the formulation by Vargaftik et al. [4], equation (3), in the slightly modified form approved by IAPWS [5].

$$
\sigma=B \tau^{\mu}(1+b \tau)
$$

In equation (3), dimensionless temperature parameter $\tau$ is given as follows

$$
\tau=1-\frac{T}{T_{\mathrm{c}}}
$$

and $B=0.2358 \mathrm{~N} \cdot \mathrm{m}^{-1}, \quad \mu=1.256, \quad b=-0.625, \quad$ and $T_{\mathrm{c}}=647.096 \mathrm{~K}$. The IAPWS correlation for surface tension [5] is valid between the triple point $0.01^{\circ} \mathrm{C}$ and temperature at the critical point $T_{\mathrm{c}}$.

Density of water both at the stable and supercooled state was evaluated from the reference equation of state IAPWS-95 [6]. IAPWS-95 provides an accurate prediction for the density also at subzero temperatures as it agrees quite well with the experimental data by Hare and Sorensen [7] measured at atmospheric pressure and temperatures between $-5{ }^{\circ} \mathrm{C}$ and $-33^{\circ} \mathrm{C}$. The uncertainty of the evaluated density is $0.03 \%$ and $0.002 \%$ at temperatures below and above $0.01{ }^{\circ} \mathrm{C}$, respectively.

The inner diameter of the glass capillary tube was calculated as an average of 17 independent measurements at various temperatures above the triple point. Its value was found to be $\bar{d}=0.3224 \pm 0.0016 \mathrm{~mm}$. Table 1 summarizes measured temperatures and heights of the water column together with the surface tension determined from equation (5).

$$
\sigma(T)=\frac{1}{4} g\left[\rho(T) h_{\mathrm{IN}}+\rho\left(T_{\mathrm{AMB}}\right) h_{\mathrm{AMB}}\right] \bar{d}
$$

The standard uncertainty of the measured surface tension was found to be below $\pm 0.39 \mathrm{mN} \cdot \mathrm{m}^{-1}$. However, the major part of the absolute uncertainty is caused by relatively high uncertainty of the IAPWS correlation [5] for the surface tension which is around $0.37 \mathrm{mN} \cdot \mathrm{m}^{-1}$ at an ambient temperature. The uncertainty of the IAPWS correlation corresponds to relative uncertainty of the evaluated surface tension of about $0.5 \%$. Excluding the uncertainty of the IAPWS correlation, the relative uncertainty of the measurement is below $0.1 \%$.

Table 1. Temperatures and heights of the water column inside a vertical capillary tube together with corresponding surface tension determined from equation (5).

\begin{tabular}{ccccc}
\hline $\begin{array}{c}\boldsymbol{T} \\
{\left[{ }^{\circ} \mathbf{C}\right]}\end{array}$ & $\begin{array}{c}\boldsymbol{T}_{\text {AMB }} \\
\left.{ }^{\circ} \mathbf{C}\right]\end{array}$ & $\begin{array}{c}\boldsymbol{h}_{\mathbf{I N}} \\
{[\mathbf{c m}]}\end{array}$ & $\begin{array}{c}\boldsymbol{h}_{\text {AMB }} \\
{[\mathbf{c m}]}\end{array}$ & $\begin{array}{c}\boldsymbol{\sigma} \\
{\left[\mathbf{m N} \cdot \mathbf{m}^{-1}\right]}\end{array}$ \\
\hline 26.26 & 25.54 & 2.885 & 6.212 & 71.70 \\
24.22 & 25.19 & 2.935 & 6.207 & 72.07 \\
22.24 & 24.62 & 2.975 & 6.200 & 72.35 \\
22.21 & 26.13 & 2.960 & 6.238 & 72.51 \\
20.31 & 25.98 & 3.016 & 6.218 & 72.80 \\
18.32 & 26.20 & 3.026 & 6.222 & 72.92 \\
16.28 & 25.37 & 2.975 & 6.310 & 73.24 \\
16.26 & 26.29 & 3.042 & 6.233 & 73.14 \\
15.06 & 24.83 & 3.121 & 6.204 & 73.57 \\
13.26 & 26.75 & 3.131 & 6.227 & 73.81 \\
13.08 & 25.43 & 3.141 & 6.209 & 73.76 \\
11.06 & 25.68 & 3.172 & 6.215 & 74.05 \\
9.05 & 25.89 & 3.207 & 6.220 & 74.37 \\
7.07 & 26.46 & 3.247 & 6.225 & 74.72 \\
5.09 & 26.07 & 3.267 & 6.230 & 74.93 \\
3.33 & 26.36 & 3.307 & 6.235 & 75.28 \\
1.40 & 26.52 & 3.317 & 6.244 & 75.43 \\
0.37 & 26.44 & 3.322 & 6.247 & 75.50 \\
-0.84 & 26.06 & 3.338 & 6.251 & 75.65 \\
-1.92 & 25.97 & 3.358 & 6.258 & 75.86 \\
-2.95 & 26.02 & 3.383 & 6.265 & 76.11 \\
-3.94 & 25.88 & 3.413 & 6.272 & 76.40 \\
-4.94 & 26.22 & 3.423 & 6.280 & 76.54 \\
-6.96 & 25.74 & 3.438 & 6.288 & 76.71 \\
-8.96 & 26.11 & 3.463 & 6.297 & 76.96 \\
-10.97 & 25.52 & 3.499 & 6.306 & 77.30 \\
\hline & & & & \\
\hline
\end{tabular}

\subsection{Comparison with other studies}

Data for the surface tension of pure water summarized in Table 1 have been compared to other experimental studies. Data for the surface tension of supercooled water have been given by Hacker [1] and Floriano and Angell [2]. Figure 5 compares all three experimental data sets together with the IAPWS correlation [5]. As can be seen, the new data agree quite well both with the data by Hacker [1] and the IAPWS correlation [5] at temperatures above the triple point. This fact confirms the validity and accuracy of the new measurement introduced in this 
study. The data by Floriano and Angell [2] are rather scattered compared to other data sources, which can be caused by a questionable temperature control of the nitrogen flow used to cool the water meniscus inside the capillary tube.

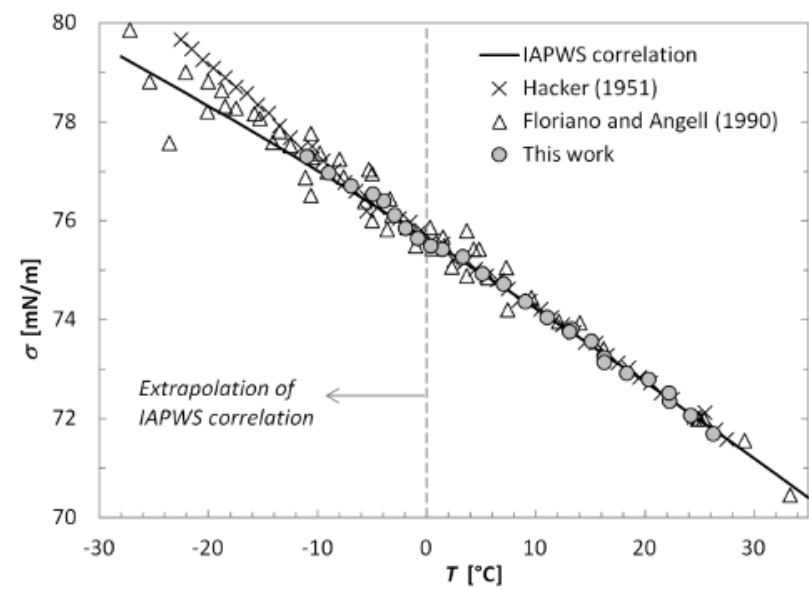

Fig. 5. Comparison of measured surface tension with other experimental data and IAPWS correlation

Figure 6 shows deviation of the experimental data for surface tension from the IAPWS correlation [5]. Unlike the data by Hacker [1], the new data for supercooled water is well approximated by the IAPWS correlation extrapolated to subzero temperatures. The scattered data by Floriano and Angell [2] have similar trend as the IAPWS correlation [5] and not as the data by Hacker [1] showing significantly higher surface tension at temperatures below $-10^{\circ} \mathrm{C}$. However further measurements at lower temperatures are necessary to prove the accuracy of the IAPWS correlation [5] extrapolated below the triple point.

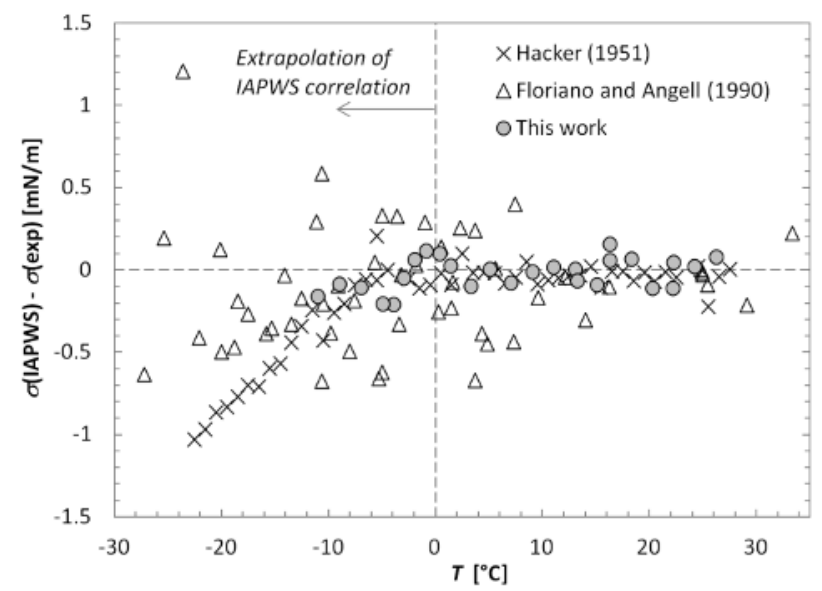

Fig. 6. Deviation of the experimental data for surface tension from the IAPWS correlation

The new experimental data does not support the existence of the second inflection point of water surface tension discussed, e.g., by Lü and Wei [8] or Kalová and Mareš [9]. The second inflection point was expected between $-10{ }^{\circ} \mathrm{C}$ and $+30{ }^{\circ} \mathrm{C}$. However, measured data do not show any discrepancy with the IAPWS correlation which does not predict any inflection point in the relevant temperature region. Accordingly, more experimental data measured in the wider temperature range are needed similarly as in case of verification of the IAPWS correlation extrapolated below the triple point.

\section{Conclusions}

A unique experimental apparatus for measurement of surface tension of supercooled aqueous systems has been developed. The functionality of the entire setup has been tested with pure water. Preliminary results for the surface tension of supercooled water have been compared with other experimental studies and with IAPWS correlation [5] which seems to provide a reasonable prediction when extrapolated below the triple point. However, more experimental data measured over wider temperature range are required to verify the accuracy of the IAPWS correlation and clarify the existence of the second inflection point of the surface tension of water.

In the next step, the authors would like to perform measurements with varying pressure of helium above the liquid meniscus inside the capillary tube. Keeping the meniscus at the defined height at all temperatures will reduce the total uncertainty of the measured surface tension. The pressure difference $\Delta p$ caused by varying surface tension will be measured directly in this case.

Measurement of surface tension in a horizontal capillary tube with pressure compensation is also under preparation. However, this goal requires still some more effort, e.g., treatment of the opened capillary tube end and tuning of the optical setup observing the flat meniscus.

\section{Acknowledgements}

The study has been supported by the grants IAA200760905 and M100761201 from the Academy of Sciences of the Czech Republic, Czech Science Foundation grants GPP101/11/P046, GAP101/11/1593, and the institutional support RVO:61388998.

\section{References}

1. P.T. Hacker, National Advisory Committee for Aeronautics, Technical Note 2510, 1-20, (1951)

2. M.A. Floriano, C.A. Angell, J. Phys. Chem. 94, 4199-4202 (1990)

3. J. Hrubý, J. Hykl, P. Peukert, B. Šmíd, EPJ Web Conf. 25, 01026 (2012)

4. N.B. Vargaftik, B.N. Volkov, L.D. Voljak, J. Phys. Chem. Ref. Data 12, 817-820 (1983)

5. IAPWS Release on Surface Tension of Ordinary Water Substance, September 1994, Accessed on 15 August 2012 (http://www.iapws.org)

6. W. Wagner, A. Pru $\beta$, J. Phys. Chem. Ref. Data 31, 387-535 (2002)

7. D.E. Hare, C.M. Sorensen, J. Chem. Phys. 87, 48404845 (1987)

8. Y.J. Lü, B. Wei, Appl. Phys. Lett. 89, 164106 (2006)

9. J. Kalová, R. Mareš, Int. J. Thermophys 33, 992-999 (2012) 